\title{
VARIATIONAL INEQUALITIES ON 2-INNER PRODUCT SPACES
}

\author{
HEDAYAT FATHI* AND SEYED ALIREZA HOSSEINIOUN
}

\begin{abstract}
We introduce variational inequality problems on 2-inner product spaces and prove several existence results for variational inequalities defined on closed convex sets. Also, the relation between variational inequality problems, best approximation problems and fixed point theory is studied.
\end{abstract}

MSC(2010): 58E35; 46B99; 47H10.

Keywords: Variational inequality, 2-Inner product spaces, Fixed point theory, Best approximation.

\section{1. introduction}

The theory of variational inequalities is an important domain of applied mathematics, introduced in the early sixties by Stampacchia and Hartman[10]. It developed rapidly because of its applications. A classical variational inequality problem is to find a vector $u^{*} \in K$ such that

$$
\left\langle v-u^{*}, T\left(u^{*}\right)\right\rangle \geq 0, \quad v \in K,
$$

where $K \subseteq \mathbb{R}^{n}$ is nonempty, closed and convex set and $T$ is a mapping from $\mathbb{R}^{n}$ into itself. Later, variational inequality expanded to Hilbert and Banach spaces. So far, a large number of existing conditions have been established. The books [11] and [3] provide a suitable introduction to variational inequality and its applications. For other generalizations of variational inequalities see [14], [15].

The concept of 2-inner product space was introduced by Diminnie, Gahler and A.White[5].

Definition 1.1. Let $X$ be a real linear space with $\operatorname{dim} X \geq 2$. Suppose that $(., . \mid$.$) is a real-valued function defined on X^{3}$ satisfying the following conditions:

(2I1) $(x, x \mid z) \geq 0$ and $(x, x \mid z)=0$ if and only if $x$ and $z$ are linearly dependent,

Date: Received: September 17, 2020, Accepted: December 6, 2020.

${ }^{*}$ Corresponding author. 
(2I2) $(x, x \mid z)=(z, z \mid x)$,

(2I3) $(y, x \mid z)=(x, y \mid z)$,

(2I4) $(\alpha x, y \mid z)=\alpha(x, y \mid z)$ for any scalar $\alpha \in \mathbb{R}$,

(2I5) $\left(x+x^{\prime}, y \mid z\right)=(x, y \mid z)+\left(x^{\prime}, y \mid z\right)$.

Then $(., . \mid$.$) is called a 2$-inner product on $X$ and $(X,(., . \mid)$.$) is called a 2$-inner product space (or 2-pre-Hilbert space).

2-inner product spaces have been intensively studied by many authors in the last three decades. A systematic presentation of the recent results related to the theory of 2-inner product spaces as well as an extensive list of the related references can be found in the book [4].

Another concept which is closely related to 2-inner product is 2-norm, which is introduced by Gahler in 1965 [9].

Definition 1.2. Let $X$ be a real linear space with $\operatorname{dim} X \geq 2$ and $\|.,$.$\| :$ $X^{2} \rightarrow[0, \infty)$ a function satisfying the following conditions for all $\alpha \in R$ and all $x, y, z \in X$ :

$(2 N 1)\|x, y\|=0 \Leftrightarrow x$ and $y$ linearly dependent,

$(2 N 2)\|x, y\|=\|y, x\|$,

(2N3) $\|\alpha x, y\|=|\alpha|\|x, y\|$,

(2N4) $\|x+y, z\| \leq\|x, z\|+\|y, z\|$,

Then $\|.,$.$\| is called 2-norm on X$ and $(X,\|.,\|$.$) is called linear 2-normed$ space.

The basic theory of 2-normed spaces can be found in [8]. Some basic properties of 2-inner product and 2-norm can be immediately obtained are as follows:

(1) $(0, y \mid z)=0,(x, 0 \mid z)=0$.

(2) For $x, y, z \in X$ we have the Couchy-Schwartz inequality

$$
|(x, y \mid z)| \leq \sqrt{(x, x \mid z)} \sqrt{(y, y \mid z)} .
$$

(3) $\|x, y\|$ is non-negative for all $x, y \in X$.

(4) $\|x, y+\alpha x\|=\|x, y\|$ for all $x, y \in X$ and $\alpha \in \mathbb{R}$.

The next theorem provides a relation between 2-norms and 2-inner products.

Theorem 1.3. [4] On any 2-inner product space $(X,(., . \mid)),.\|a, b\|=\sqrt{(a, a \mid b)}$ defines a 2-norm for which

$$
(a, b \mid c)=\frac{1}{4}\left(\|a+b, c\|^{2}-\|a-b, c\|^{2}\right)
$$

and (the 2-dimensional analogue of the Parallelogram Law)

$$
\|a+b, c\|^{2}+\|a-b, c\|^{2}=2\left(\|a, c\|^{2}+\|b, c\|^{2}\right) .
$$

Conversely if Parallelogram Law established in linear 2-normed space $(X,\|.,\|$.$) then (1.1)$ defines a 2 -inner product on $X$.

Whenever a 2 -inner product space $(X,(., .)$.$) is given, we consider it as a$ linear 2-normed space $(X,\|.,\|$.$) with the 2-norm defined in Theorem (1.3).$ 
For a fixed $z \in X$, the function $P_{z}(x)=\|x, z\|(x \in X)$, is a seminorm on $X$. Since $\operatorname{dim} X>1$ for any $y \neq 0$ there exists $x \in X$ such that $x$ and $y$ are linearly independent and hence $P_{x}(y) \neq 0$. So the class $P=\left\{P_{z}: z \in X\right\}$ forms a family of semi-norms which separates points in $X$ and generates a locally convex topology on $X$, which is called the natural topology induced by the 2 -norm $\|.,$.$\| .$

Definition 1.4. Let $(X,\|.,\|$.$) be a 2-normed space, and u \in X$.

(1) A sequence $\left\{x_{n}\right\} \subseteq X$ is said to be $u$-convergent to $x \in X$ and denoted by $x_{n} \stackrel{u}{\longrightarrow} x$, if $\lim _{n \rightarrow \infty}\left\|x_{n}-x, u\right\|=0$.

(2) A subset $K$ of $X$ is said $u$-closed, if $\left\{x_{n}\right\} \subseteq K$ is $u$-convergent to $x \in X$, then $x \in K$.

\section{Variational inequalities on 2-inner product spaces}

In this section, we give the natural generalization of variational inequality on 2-inner product spaces and then we prove our main theorem.

Definition 2.1. Let $K$ be an arbitrary nonempty subset of real 2-inner product space $X, 0 \neq u \in X$ and $T: K \longmapsto X$ be a mapping. The set of variational inequality corresponds to $T, K$, and $u$, denoted by $V I(T, K, u)$, is the set of all $x_{0} \in K$ that apply to the following condition

$$
\left(x-x_{0}, T\left(x_{0}\right) \mid u\right) \geq 0 \quad \forall x \in K .
$$

Some immediate consequences of definition 2.1 are listed below:

(1) If there exists $x_{0}$ such that $T\left(x_{0}\right)=\alpha u$ for some $\alpha \in \mathbb{R}$ then $x_{0}$ is a solution of $\operatorname{VI}(T, K, u)$.

(2) If $u \in K$ and $x_{0} \in K$ is a solution of $V I(T, K, u)$, then $\left(x_{0}, T\left(x_{0}\right) \mid u\right) \leq$ 0 .

Before proving existence theorems we recall the following notions and prove analog of a Mintys lemma [13].

Definition 2.2. Let $K \subseteq X$ be a closed and convex set, $T: K \longmapsto X$ be a mapping and $u \in X$

(i) $T$ is said to be $u$-monotone if

$$
(x-y, T(x)-T(y) \mid u) \geq 0 \quad(x, y \in K) .
$$

(iii) $T$ is said to be strictly $u$-monotone if $x \neq y$ implies that

$$
(x-y, T(x)-T(y) \mid u)>0 .
$$

(v) $T$ is said to be $u$-pseudo monotone if $(x-y, T(y) \mid u) \geq 0$ implies $(x-y, T(x) \mid u) \geq 0$. 
Lemma 2.3. Let $u \in X, K \subseteq X$ be a closed and convex set and $T: K \longmapsto X$ be a continuous and $u$-pseudo monotone. Then an element $x_{0} \in K$ is a solution of $\operatorname{VI}(T, K, u)$ if and only if

$$
\left(x-x_{0}, T(x) \mid u\right) \geq 0 \quad \forall x \in K .
$$

Proof. Suppose that $x_{0} \in K$ is a solution of $V I(T, K, u)$. Then for any $x \in K$, we have $\left(x-x_{0}, T\left(x_{0}\right) \mid u\right) \geq 0$ and the $u$-pseudo monotonicity implies that $\left(x-x_{0}, T(x) \mid u\right) \geq 0$.

Conversely, suppose $x_{0} \in K$ holds in condition (2.1). In this case, if $x \in K$, we define $x_{t}$ by

$$
x_{t}=(1-t) x_{0}+t x, \quad t \in(0,1) .
$$

Insert $x_{t}$ in (2.1), we have

$$
\left(x_{t}-x_{0}, T\left(x_{t}\right) \mid u\right) \geq 0
$$

which implies that

$$
\left(t\left(x_{t}-x_{0}\right), T\left(x_{t}\right) \mid u\right) \geq 0
$$

and finally

$$
\left(x-x_{0}, T\left(x_{t}\right) \mid u\right) \geq 0 .
$$

By taking the limit in the above inequality and by using the continuity of $T$, we get

$$
\left(x-x_{0}, T\left(x_{0}\right) \mid u\right) \geq 0 .
$$

i.e. $x_{0}$ is a solution of $V I(T, K, u)$.

Remark 2.4. The set $V I(T, K, u)$ is not necessarily single-valued. But when $T$ is $u$-strictly monotone the uniqueness property holds. In fact if $x, x^{\prime} \in K$ are two solutions of $\operatorname{VI}(T, K, u)$ then

$$
\begin{gathered}
(y-x, T(x) \mid u) \geq 0 \quad y \in K, \\
\left(y-x^{\prime}, T\left(x^{\prime}\right) \mid u\right) \geq 0 \quad y \in K .
\end{gathered}
$$

So, setting $y=x^{\prime}$ in first inequality and $y=x$ in second, we have

$$
\left(x-x^{\prime}, T(x)-T\left(x^{\prime}\right) \mid u\right) \leq 0 .
$$

Now strictly monotonicity implies that $x=x^{\prime}$.

For any subset $S$ of $X$ define $u$-orthogonal complement of $S$ by

$$
S_{u}^{\perp}=\{x \in X \mid(x, y \mid u)=0, \forall y \in S\} .
$$

Proposition 2.5. Let $S \subseteq X$ be nonempty and closed under scaler multiplication, $u \in X$ and $T: S \rightarrow X$ be a mapping. Then $x_{0}$ is solution to $V I(T, S, u)$ if and only if $T\left(x_{0}\right) \in S_{u}^{\perp}$.

Proof. If $x_{0}$ is a solution of $V I(T, S, u)$ and $x \in S$ then $\left(x-x_{0}, T\left(x_{0}\right) \mid u\right) \geq 0$. Now $0,-x \in s$ implies that $\left(x, T\left(x_{0}\right) \mid u\right)=0$. Conversely if $T\left(x_{0}\right) \in S_{u}^{\perp}$ then clearly $\left(x-x_{0}, T\left(x_{0}\right) \mid u\right)=0$ and the proof is complete.

The following theorem is fundamental existence theorem for $V I(T, K, u)$. 
Theorem 2.6. Let $K$ be a compact convex subset of $X, u \in X$ and $T: K \longrightarrow$ $X$ be continuous, then $V I(T, K, u)$ is nonempty.

Proof. It is well known that in inner product space $(X,\langle.,\rangle$.$) if K \subseteq X$ is compact and convex and $T: K \longrightarrow X$ is continuous, then $V I(T, K)$ has a solution. An easy computation shows that, if $u, v \in X$ is linearly independent then for every $t \in(0,1]$,

$$
\langle x, y\rangle_{t}=(x, y \mid u)+(x, y \mid t v) \quad(x, y \in X)
$$

is an inner product on $X$. So there exists $x_{t} \in K$ such that

$$
\left\langle x-x_{t}, T\left(x_{t}\right)\right\rangle_{t} \geq 0, \quad(x \in K) .
$$

In other word

$$
\left(x-x_{t}, T\left(x_{t}\right) \mid u\right)+\left(x-x_{t}, T\left(x_{t}\right) \mid t v\right) \geq 0 \quad(x \in K) .
$$

Since $K$ is compact, then $\left\{x_{t}\right\}_{t}$ has a limit point $x_{0} \in K$. Now continuity of $T$ implies that

$$
\left(x-x_{0}, T\left(x_{0}\right) \mid u\right) \geq 0, \quad(x \in K) .
$$

Hence $x_{0} \in V I(T, K, u)$ and this completes the proof.

In general, if we have a monotonicity assumption then by the well-known KKM-Fan lemma, we can prove an existence result for closed, bounded and convex set. For the main result of this paper, we need the following famous lemma of $K-$ Fan.

Lemma 2.7. [7] Let $K$ be a nonempty, closed and convex subset of a Hausdorff topological space and $S: K \rightarrow 2^{K}$ be a multivalued map. Suppose that for any finite set $\left\{x_{1}, \cdots x_{n}\right\} \subseteq K$ one has $\operatorname{conv}\left\{x_{1}, \cdots x_{n}\right\} \subseteq \bigcup_{i=1}^{n} S\left(x_{i}\right)$, (i.e., $S$ is a KKM-mapping) and $S(x)$ is closed for each $x \in K$ and compact for some $x \in K$, where conv denotes the convex hull operator. Then $\bigcap_{x \in K} S(x) \neq \emptyset$.

Theorem 2.8. Let $K$ be a closed, bounded and convex subset of $X, u \in X$ and $T: K \longrightarrow X$ be $u$-pseudo monotone and continuous and there exists $x \in K$ such that the set $\{y \in K:(x-y, T(x) \mid u) \geq 0\}$ is weakly compact, then $V I(T, K, u)$ has a solution.

Proof. Let $x_{1}, \cdots, x_{n} \in E, M=\mathbb{C} x_{1}+\cdots+\mathbb{C} x_{n}$ and $K_{M}=M \bigcap K$. Then $K_{M}$ is a compact and convex subset of $M$ and $T$ is continuous on $K_{M}$. Thus by Theorem 2.6, $\operatorname{VI}\left(T, K_{M}, u\right)$ has a solution, say $v_{M}$. By Lemma 2.3

$$
\left(x-v_{m}, T(x) \mid u\right) \geq 0 \quad\left(x \in K_{M}\right) .
$$

Define

$$
S(x)=\{y \in K:(x-y, T(x) \mid u) \geq 0\} .
$$

$S(x)$ is nonempty ( it is enough to set $M=\mathbb{C} x$ ), convex, closed and elements of $\bigcap_{x \in K} S(x)$ will be the solutions of $V I(T, K, u)$. It is enough to show that $\bigcap_{x \in K} S(x)$ is nonempty. To prove this fact, we use Lemma ??. 
Let $\left\{x_{1}, \cdots, x_{m}\right\} \subseteq K$. We claim that $S$ is KKM mapping. If it is not the case, then there exists a finite set $\left\{y_{1}, \cdots, y_{m}\right\} \subseteq K$ and $t_{i} \geq 0, i=1, \cdots, n$ such that

$$
y=\sum_{i=1}^{n} t_{i} y_{i} \notin \bigcup_{i=1}^{n} S\left(x_{i}\right), \quad \sum_{i=1}^{n} t_{i}=1 .
$$

So

$$
\left(y_{i}-y, T\left(y_{i}\right) \mid u\right)<0, \quad 0, i=1, \cdots, n
$$

Which implies

$$
\left(t_{i} y_{i}-t_{i} y, t_{i} T\left(y_{i}\right) \mid u\right)<0, \quad 0, i=1, \cdots, n .
$$

Now we have by addition

$$
0>\left(\sum_{i=1}^{n} t_{i} y_{i}-y, T\left(\sum_{i=1}^{n} t_{i} y_{i}\right)=(y-y, T(y) \mid u)=0 .\right.
$$

This is a contradiction. So $S$ is a KKM-mapping and this completes the proof.

Remark 2.9. In the above theorem, if $X$ is reflexive (as a locally convex space) then $S(x)$ is weakly closed for every $x \in K$ and the Banach-Alauglo theorem implies that $S(x)$ is weakly compact. So, if $X$ is reflexive then Theorem 2.6 is satisfied for bounded closed convex sets.

In the two next theorems, we consider unbounded closed convex sets.

Theorem 2.10. Let $T$ and $u$ be as the previous theorem and $K$ be a closed convex subset of $X$. A necessary and sufficient condition for the set $\mathrm{VI}(\mathrm{T}, \mathrm{K}, \mathrm{u})$ to be nonempty is that:

for some positive real number $R$ there exists a solution $x_{R}$ of the variational inequality $V I\left(T, K_{R}, u\right)$ where $\quad\left(K_{R}=K \bigcap\{v:\|v, u\| \leq R\}\right)$, satisfies the inequality $\left\|x_{R}, u\right\|<R$.

Proof. Let $x_{0}$ be a solution of $V I(T, K, u)$. Choose $R>0$ such that $\left\|x_{0}, u\right\|<R$. Then $x_{0}$ is a solution of $V I\left(T, K_{R}, u\right)$ as required. Conversely, If $x_{R} \in K_{R}$ is a solution of $V I\left(T, K_{R}, u\right)$ and $\left\|x_{R}, u\right\|<R$. then $x_{R}$ is an inner point of $K_{R}$ in the semi normed space $\left(X, P_{u}\right)$. Thus there exists $\epsilon>0$ such that $N_{\epsilon}\left(x_{R}\right) \subseteq K_{R}$. If $y \in K$ is arbitrary then $\frac{\epsilon\left(y-x_{R}\right)}{2\left\|y-x_{R}, u\right\|}+x_{R} \epsilon$ $N_{\epsilon}\left(x_{R}\right) \subseteq K_{R}$. So $\left(y-x_{R}, T\left(x_{R}\right) \mid u\right) \geq 0$.

Definition 2.11. A mapping $T: K \longrightarrow X$ is called $u$-coercive on $K$ if there exists an element $x_{0} \in K$ such that

$$
\frac{\left(x-x_{0}, T x-T x_{0} \mid u\right)}{\left\|x-x_{0}, u\right\|} \longrightarrow+\infty, \text { as }\|x, u\| \longrightarrow \infty, \quad x \in K .
$$

Theorem 2.12. Let $K \subseteq X$ be a nonempty closed and convex set and $T$ : $K \longrightarrow X$ be monotone, $u$-coercive and continuous. Then $V I(T, K, u)$ is nonempty. 
Proof. If we prove that there exists a solution $x_{R}$ for $V I\left(T, K_{R}, u\right)$ such that $\left\|x_{R}, u\right\|<R$. By choosing $r>\left|T\left(x_{0}\right)\right|$ and $R>\left\|x_{0}, u\right\|$ such that $\|x, u\| \geq R$ we can conclude that

$$
\left(x-x_{0}, T x-T x_{0} \mid u\right) \geq r\left\|x-x_{0}, u\right\| .
$$

Now if $\|x, u\|=R$ then we have

$$
\begin{aligned}
\left(x-x_{0}, T x \mid u\right) & \geq r\left\|x-x_{0}\right\|+\left(x-x_{0}, T x_{0} \mid u\right) \\
& \geq r\left\|x-x_{0}\right\|-\left\|T x_{0}, u\right\|\left\|x-x_{0}, u\right\| \\
& \geq\left(r-\left\|T x_{0}, u\right\|\right)\left\|x-x_{0}, u\right\|>0 .
\end{aligned}
$$

Let $x_{R} \in K_{R}$ be a solution of $V I\left(T, K_{R}, u\right)$. Then

$$
\left(x_{R}-x_{0}, T x_{R} \mid u\right)=-\left(x_{0}-x_{R}, T x_{R} \mid u\right) \leq 0 .
$$

So $\left\|x_{R}, u\right\| \neq R$. In other words $\left\|x_{R}, u\right\|<R$.

Proposition 2.13. Let $x$ be a solution of $V I(T, K \mid u)$ and suppose that $x$ is an interior point of $K$ in semi normed space $\left(X, P_{u}\right)$. Then $T(x)=0$.

Proof. $x_{0} \in K^{\circ}$ implies that there exists an $\epsilon>0$ such that $N_{\epsilon}\left(x_{0}\right) \subseteq K$. So if $x \in X$ is arbitrary then $\frac{\epsilon x}{2\|x, u\|}+x_{0} \in N_{\epsilon}\left(x_{0}\right) \subseteq K$. Which implies that $\left(x, T\left(x_{0}\right) \mid u\right) \geq 0$. Since $x$ is arbitrary, we have $T\left(x_{0}\right)=0$.

\section{3. metric projection, fixed point and variational inequalities}

The fixed point theory plays important role in variational inequalities. In this section, we consider the relationship between metric projection, fixed point and variational inequalities.

We know that, in any Hilbert space $H$, if $K$ is a closed convex subset of $H$, then there exists a unique element $P_{K}(x)$ of $K$ such that

$$
\left\|x-P_{K}(x)\right\|=\inf _{y \in K}\|x-y\| .
$$

$P_{K}(x)$ is the projection of $x$ on $K$ (or best approximation to $x$ from $K$ ), where the mapping $P_{K}: H \longrightarrow K$ is the metric projection onto $K$.

Recently, many works on best approximation have been done in 2-normed and 2-inner product spaces(see for example, [1], [2], [6], [16] and the references therein.)

Definition 3.1. [16] Let $(X,\|.,\|$.$) be a 2-normed space, K$ a subset of $X$, $u \in X$ and $\langle u\rangle$ the subspace of $X$ generated by $u . x_{0} \in K$ is called a $u$-best approximation of $x \in K$, if

$$
\left\|x-x_{0}, u\right\|=\inf _{y \in K}\|x-y, u\| .
$$

The set of all $u$-best approximations of $x$ in $K$ is denoted by $P_{K}^{u}$. $K$ is called $u$-proximinal(resp. $u$-Chebyshev) if for every $x \in X \backslash(K+\langle u\rangle)$, there exists at least (resp. exactly) one $u$-best approximation in $K$. 
It is well known that every nonempty $u$-closed and convex set in a $u$ Hilbert space is $u$-chebyshev.(see for example [12]). Some properties of $p_{K}^{u}$ (for example $u$-monotones, uniformly continuity and etc.) are collected in proposition 3.1. of [1]. Also in [18] Theorem 2.7. it is shown that if $(X,(., . \mid)$. is a 2-inner product space, $x, u \in X, K \subseteq X$ is closed and convex, then $p=P_{K}^{u}(x)$ if and only if $p \in K$ and $(x-p, p-y \mid u) \geq 0 \quad(y \in K)$. So $P_{K}^{u}(x)$ is characterized by a variational inequality. In fact, if $T: y \mapsto y-x, \quad(x \in K)$, then $p=P_{K}^{u}(x)$ if and only if $p$ is the solution of $V I(T, K, u)$. Thus finding $P_{K}^{u}(x)$ for any $x \in E$ is a variational problem and $P_{K}^{u}$ has monotonicity and continuity properties that we need. Hence, we can apply the main theorems of this paper to find $P_{K}^{u}(x)$.

Here we give another proof of (Theorem 2.3. [12]).

Corollary 3.2. If $(X,(., . \mid)$.$) is a 2-inner product space and K \subseteq X$ is a closed and convex set then $P_{K}^{u}(x)$ has at most one element.

Proof. Since the map $T: y \mapsto y-x, \quad(y \in K)$ is $u$-strictly monotone, Remark 2.4 proofs the corollary.

In the next two propositions, by using the best approximation, we prove equivalent statements for the solvability of variational inequalities.

Proposition 3.3. Let $K \subseteq X$ be closed convex, $u \in X$ and $T: K \longrightarrow X$ be a mapping. Then $x_{0}$ is a solution of $V I(T, K, u)$ if and only if $x_{0}$ is a fixed point of the map $P_{K}^{u}(I-\rho T): K \longrightarrow K$. That is $x_{0}=P_{K}^{u}\left(x_{0}-\rho T\left(x_{0}\right)\right)$, where $\rho>0$ is constant.

Proof. Let $x \in K$ and $x_{0}$ be a solution of $V I(T, K, u)$. Thus $\left(x-x_{0}, \rho T\left(x_{0}\right) \mid u\right) \geq$ 0 or equivalently $\left(x-x_{0}, x_{0}+\rho T\left(x_{0}\right)-x_{0} \mid u\right) \geq 0$. Now variational characterization of best approximation implies that $x_{0}=P_{K}^{u}\left(x_{0}-\rho T\left(x_{0}\right)\right)$.

Conversely, if $x_{0}=P_{K}^{u}\left(x_{0}-\rho T\left(x_{0}\right)\right)$ then $\left(x_{0}-x, x_{0}+\rho T\left(x_{0}\right)-x_{0} \mid u\right) \geq 0$. Hence $\left(x_{0}-x, T\left(x_{0}\right) \mid u\right) \geq 0$. That is $x_{0}$ is a solution of $V I(T, K, u)$.

Definition 3.4. Let $K$ be a closed and convex subset of $X$. The operator $T_{K}: X \rightarrow X$ defined by $T_{K}(z)=T\left(P_{K}^{u}(z)\right)+z-P_{K}^{u}(z) \quad(z \in X)$ is called the normal operator associated with $T, K$ and u.[17]

Proposition 3.5. An element $x_{0} \in X$ is a solution of the equation $T_{K}\left(x_{0}\right)=0$ if and only if $p=P_{K}^{u}\left(x_{0}\right)$ is a solution of the $V I(T, K, u)$.

Proof. If $T_{K}\left(x_{0}\right)=0$ then we have $T(p)+x_{0}-p=0$. On the other hand,

$$
\left(x_{0}-p, p-x \mid u\right) \geq 0 \quad(x \in K) .
$$

So we have $(x-p, T(p) \mid u) \geq 0$.

Conversely, if $x \in K, x_{0}=p-T(p)$ and $(x-p, T(p) \mid u) \geq 0$, we have $T(p)=p-x_{0}$. So $\left(x-p, p-x_{0} \mid u\right) \geq 0$. Which implies that $p=P_{K}^{u}\left(x_{0}\right)$. Therefore we have $T_{K}\left(x_{0}\right)=0$. and this completes the proof.

The next theorem is an existence theorem based on Banach fixed point theorem. 
Theorem 3.6. Let $K$ be a nonempty closed convex $u$-chebyshev subset of $X$ and $T: K \rightarrow E$ an operator satisfying

$$
\begin{aligned}
(T(x)-T(y), x-y \mid u) \geq m\|x-y, u\|^{2} & (x, y \in K), \\
\|T(x)-T(y), u\| \leq M\|x-y, u\| & (x, y \in K),
\end{aligned}
$$

where, $m$ and $M$ are positive constants. Then there exists a unique solution for $V I(T, K, u)$.

Proof. We show that if the number $\rho$ is chosen properly $U=P_{K}^{u}(I-\rho T)$ For every $x, y \in X$ is a contraction mapping then Proposition 3.3 will proves the theorem.

$$
\begin{aligned}
\|U(x)-U(y), u\|^{2} & \leq\|(I-\rho T)(x)-(I-\rho T)(y), u\|^{2} \\
& =(x-\rho T(x)-y+\rho T(y), x-\rho T(x)-y+\rho T(y) \mid u) \\
& =\|x-y, u\|^{2}-2 \rho(T(x)-T(y), x-y \mid u)+\rho^{2}\|T(x)-T(y), u\|^{2} \\
& \leq\left(1-2 \rho m+\rho^{2} M^{2}\right)\|x-y, u\|^{2} .
\end{aligned}
$$

Thus

$$
\|U(x)-U(y)\|^{2} \leq\left|1-2 \rho m+\rho^{2} M^{2}\right|\|x-y, u\|^{2} .
$$

Now if we suppose that $\rho$ is small enough, then $U$ will be a contraction. Since we can always choose $\rho$ so, $U$ can always be constructed so. Hence there exists a unique solution to the $\operatorname{VI}(T, K, u)$.

More ever, proof of the above theorem shows that, the solution of $\operatorname{VI}(T, K, u)$ can be obtained as the limit of the sequence generated by the classical iterative process

whenever $0<\rho<\frac{2 m}{M^{2}}$.

$$
x^{n+1}=U\left(x^{n+1}\right)=P_{K}^{u}\left(x^{n}-\rho T\left(x^{n}\right)\right)
$$

\section{REFERENCES}

[1] M. Abrishami-Moghaddam, T. Sistani, Best approximation for convex subsets of 2inner product spaces, Journal of Nonlinear Analysis and Optimization, 3(2): pp. 269-278, (2012).

[2] M. Acikgoz, Some results best and 2-best approximation on 2-structures, Mathematica Morica, 13: pp. 1-11, (2009).

[3] C. Baiocchi and A. capello, variational and quasi-variational Inequalities. Applications to free boundary problems, J. wily and sons, New York, 1984.

[4] Y.J. Cho, C.S. Lin, S.S. Kim and A. Misiak, Theory of 2-Inner Product Spaces, Nova Science Publishers, Inc., New York, 2001.

[5] C. Diminnie, S. Gahler and A.White, 2-inner product spaces, Demonstratio Math. 6: 525-536, (1973).

[6] S. Elumalai, R. Vijayaragavan, Characterizations of best approximations in linear 2-normed spaces, General Mathematics Vol. 17(1): pp. 141-160, (2009).

[7] K. Fan, Some properties of convex sets to fixed point theorem, Math. Ann. 266: pp. 519-537, (1984).

[8] R.W. Freese and Y.J. Cho, Geometry of Linear 2-Normed Spaces, Nova Science Publishers, Inc., New York, 2001. 
[9] S. Gahler, Lineare 2-normierte raume. Math. Nachr. 28:: 1-43, (1964).

[10] Ph. Hartman and G. Stampacchia, On some nonlinear elliptic differential functional equations, Acta math. 115: pp. 271-310, (1966).

[11] D. Kinderlehrer and G. Stampacchia, An Introduction to Variational Inequalities and Their Applications, Academic press, New York, 1980.

[12] H. Mazaheri, R. Kazemi, Some results on 2-inner product spaces, Novi Sad J. Math., 37(2): pp. 35-40, (2007).

[13] G.J. Minty, Monotone (nonlinear) operators in Hilbert space, Duke Math. 29, pp.341346, (1964).

[14] M.A. Noor, Generel variational inequalities, Appl. Math. lett. 1: pp. 119-121, (1988).

[15] M.A. Noor, Extended general variational inequalities, Appl. Math. lett. 22: pp. 182186, (2009).

[16] Sh. Rezapour, Proximinal subspaces of 2-normed spaces, Ana. Theory Appl. 22(2): pp. 114-119, (2006).

[17] S.M. Robinson, Nonsingularity and symmetry for linear normal maps, Math. Programming, 62: pp. 415-425, (1993).

[18] T. Sistani, M. Abrishami-Moghaddam, Some results on best approximation in convex subsets of 2-normed spaces, Int.J.Math.analysis,21(3): pp. 1043-1049, (2009).

(Hedayat Fathi) Department of Mathematics, Shahid Beheshti University, TEHRAN, Iran

Email address: Hedayat.fatthi@gmail.com

(Seyed Alireza Hosseinioun) Department of Mathematics, Shahid Beheshti UniVERSITY, TEHRAN, IRAN

Email address: ahosseinioun@yahoo.com 